\title{
CHEMICAL HOMOGENEITY IN THE HYADES
}

\author{
G. M. De Silva, ${ }^{1}$ C. Sneden, ${ }^{2}$ D. B. Paulson, ${ }^{2}$ M. Asplund, ${ }^{1}$ J. Bland-Hawthorn, ${ }^{3}$ \\ M. S. Bessell, ${ }^{1}$ and K. C. Freeman ${ }^{1}$ \\ Received 2005 May 25; accepted 2005 September 8
}

\begin{abstract}
We present an abundance analysis of the heavy elements $\mathrm{Zr}$, Ba, La, Ce, and $\mathrm{Nd}$ for Hyades $\mathrm{F}-\mathrm{K}$ dwarfs based on high-resolution, high signal-to-noise ratio spectra from Keck HIRES. The derived abundances show the stellar members to be highly uniform, although some elements show a small residual trend with temperature. The rms scatter for each element for the cluster members is as follows: $\mathrm{Zr}=0.055, \mathrm{Ba}=0.049, \mathrm{Ce}=0.025, \mathrm{La}=0.025$, and $\mathrm{Nd}=0.032 \mathrm{dex}$. This is consistent with the measurement errors and implies that there is little or no intrinsic scatter among the Hyades members. Several stars thought to be nonmembers of the cluster based on their kinematics are found to deviate from the cluster mean abundances by about $2 \sigma$. Establishing chemical homogeneity in open clusters is the primary requirement for the viability of chemically tagging Galactic disk stars to common formation sites in order to unravel the dissipative history of early disk formation.
\end{abstract}

Key words: open clusters and associations: individual (Hyades) — stars: abundances

\section{INTRODUCTION}

One of the major goals of near-field cosmology is to determine the sequence of events involved in the formation of the Galactic disk during the epoch of dissipation. Since the disk formed dissipatively and evolved dynamically, most of the dynamical information is lost. However, locked away within the stars, the chemical information survives. If star-forming aggregates have unique chemical signatures, we can use the method of chemical tagging to track individual stars back to a common formation site (Freeman \& Bland-Hawthorn 2002). With sufficiently detailed abundances we would be able to reconstruct the stellar aggregates that have long since diffused into the Galaxy background. The critical issue for the viability of this method is whether the star-forming aggregates do indeed have unique chemical signatures. The essential first step is to investigate the chemical homogeneity of open clusters, which are the likely leftovers of star-forming aggregates in the Galactic disk.

Chemical homogeneity in an open cluster implies that the progenitor cloud was uniformly mixed before its stars formed. Theoretical work on star formation in giant molecular clouds suggests the presence of high levels of turbulence (McKee \& Tan 2002), which would result in a well-mixed gas cloud. However, it is not clear whether this mixing occurs during cloud collapse (before the birth of the first stars) or whether a few high-mass stars form first, shortly after the cloud assembles, and then enrich the cloud uniformly. With both scenarios, however, one expects to find homogeneity among the long-lived stars in certain key elements.

The heavier neutron-capture elements are the key to establishing chemical homogeneity, as their abundances are not thought to be modified during normal stellar evolution. The $s$-process elements are believed to arise from the He-burning phase of asymptotic giant branch stars, while the most likely site for $r$-process elements appears to be Type II supernovae (Burbidge et al. 1957; Wallerstein et al. 1997). As a result, these heavier elements (along

\footnotetext{
${ }^{1}$ Mount Stromlo Observatory, Australian National University, Cotter Road, Weston, ACT 2611, Australia; gayandhi@mso.anu.edu.au.

2 Department of Astronomy, University of Texas, Austin, TX 78712; chris@ verdi.as.utexas.edu.

3 Anglo-Australian Observatory, Eastwood, NSW 2122, Australia; jbh@ aao.gov.au.
}

with some $\alpha$ and Fe-peak elements) are more representative of the conditions of the progenitor cloud from which the cluster formed. Furthermore, the neutron-capture elements are particularly useful for identifying chemical signatures due to the large intrinsic (cosmic) scatter in their abundances (Edvardsson et al. 1993; Reddy et al. 2003), in particular at low [Fe/H].

Published observations of both light- and heavy-element abundances in open clusters demonstrate chemical homogeneity, albeit for only a few stars, and lend support to the prospect of chemical tagging. Some recent examples include Friel et al. (2003), who studied the $\alpha$-elements for four giants in the old open cluster Collinder 261 and found $1 \sigma$ dispersions about the mean, consistent with the expected uncertainties. Schuler et al. (2003) obtained chemical homogeneity for several elements over nine stars in M34, except for K, which has a tightly correlated temperature trend thought to be due to systematic effects in the model atmospheres. Gonzalez \& Wallerstein (2000) found homogeneity in Eu for four stars in M11, although there was significant scatter in the $\alpha$-elements. Tautvaišiene et al. (2000) studied nine stars in M67 and found the heavy-element abundances to be almost identical in all stars. Castro et al. (1999) observed that the $[\mathrm{Ba} / \mathrm{Fe}]$ ratios in the Ursa Major moving group stand out in comparison with solar neighborhood normal stars with the same metallicity.

We begin our study on chemical homogeneity with the Hyades. It is the nearest cluster to the Sun, with an age of $\approx 625 \mathrm{Myr}$ (Perryman et al. 1998) and $[\mathrm{Fe} / \mathrm{H}] \approx 0.13$ (Paulson et al. 2003, hereafter P03). It has been extensively studied in the past, with stellar memberships firmly established (de Bruijne et al. 2001; Hoogerwerf \& Aguilar 1999). The Hyades stars have been subject to numerous abundance studies in past years, from Conti et al. (1965) to, most recently, P03. However, to the present date there has been little work done on the abundance analysis of the heavier neutron-capture elements for the Hyades dwarfs. Therefore, the present results for heavy-element abundances over a large sample of Hyades dwarfs are the first of their kind.

\section{CHEMICAL ABUNDANCES}

\subsection{Observational Data}

This study of the neutron-capture elements uses a subset of the data sample analyzed by $\mathrm{P} 03$. The original observations were 
TABLE 1

LiNE LisT

\begin{tabular}{|c|c|c|c|c|}
\hline Element & $\begin{array}{l}\text { Wavelength } \\
\text { (Å) }\end{array}$ & $\begin{array}{c}\chi \\
(\mathrm{eV})\end{array}$ & $\log g f$ & References \\
\hline Zr II................. & 4050.33 & 0.710 & -1.000 & 1 \\
\hline Ва II ................... & 4130.57 & 2.722 & 0.680 & 1 \\
\hline Ва II ................. & 4554.04 & 0.000 & 0.170 & 1 \\
\hline 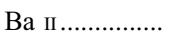 & 4934.09 & 0.000 & -0.150 & 1 \\
\hline Ва II .................. & 5853.69 & 0.604 & -1.000 & 1 \\
\hline Ва II .................... & 6141.73 & 0.704 & -0.076 & 1 \\
\hline La II ..................... & 3988.52 & 0.400 & 0.210 & 2 \\
\hline La II ..................... & 3995.75 & 0.170 & -0.060 & 2 \\
\hline 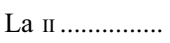 & 4086.71 & 0.000 & -0.070 & 2 \\
\hline La II .................... & 4662.50 & 0.173 & -1.240 & 2 \\
\hline Ce II .................... & 4083.22 & 0.701 & 0.270 & 3 \\
\hline 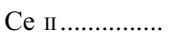 & 4364.65 & 0.495 & -0.230 & 3 \\
\hline 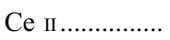 & 4562.36 & 0.478 & 0.230 & 3 \\
\hline $\mathrm{Ce}$ II .................... & 4628.16 & 0.516 & 0.200 & 3 \\
\hline $\mathrm{Nd}$ II .............. & 4012.24 & 0.630 & 0.810 & 4 \\
\hline Nd II ................ & 4012.69 & 0.000 & -0.600 & 4 \\
\hline $\mathrm{Nd}$ II .................. & 4018.82 & 0.063 & -0.850 & 4 \\
\hline Nd II ............... & 4068.89 & 0.000 & -1.420 & 4 \\
\hline Nd II .................. & 4462.98 & 0.559 & 0.040 & 4 \\
\hline
\end{tabular}

References. - (1) Vienna Atomic Line Database (Kupka et al. 1999; Ryabchikova et al. 1999; Piskunov et al. 1995); (2) Lawler et al. 2001; (3) Palmeri et al. 2000; (4) Den Hartog et al. 2003.

part of the planet search program undertaken with the Keck I HIRES from 1996 to 2000. A full description of the observations can be found in Cochran et al. (2002). Those stars with higher rotation $\left(v \sin i>10 \mathrm{~km} \mathrm{~s}^{-1}\right)$ were left out of this study due to the poorer spectral resolution and blended lines making it difficult to identify the weak lines of the heavier elements of interest.

The signal-to-noise ratio of each spectrum is typically 100 $200 \mathrm{pixel}^{-1}$, and resolving power is nominally at 60,000 . The spectra cover the wavelength region from 3800 to $6200 \AA$, which is an ideal region for the majority of the neutron-capture lines (Bland-Hawthorn \& Freeman 2004). Details of the preliminary data reduction procedures can be found in P03.

\subsection{Model Atmospheres and Spectral Lines}

Interpolated Kurucz model atmospheres based on the ATLAS9 code (Castelli et al. 1997) with no convective overshoot were used for this study. Spectral lines were selected in comparison to the solar atlas (Beckers et al. 1976). Preference was given to clean, weaker lines covering a range of excitation potentials. All lines that are formed on or near the wings of other lines were discarded, as well as any lines with significant blending. Most of the lines used in this analysis have some degree of blending due to the crowded spectral region in which most of the neutroncapture element lines are formed. The full list of lines and references used for this analysis is given in Table 1.

\subsection{Abundance Analysis}

Details of the stellar parameter analysis are described in P03. The same parameters have been used for this study, as we are using the same data set.

The abundance analysis makes use of the MOOG code (Sneden 1973) for LTE equivalent width (EW) analysis and spectral syntheses. Depending on the degree of blending of the spectral lines, abundances were derived either by EW measurements or by spectral synthesis. The EWs were measured by fitting a

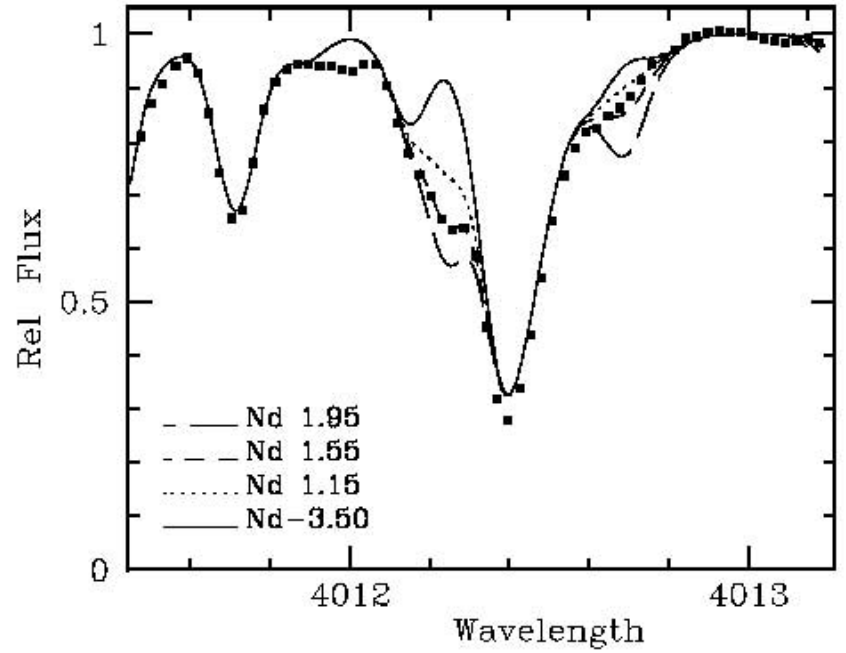

Fig. 1.- Sample synthetic (dashed and dotted curves) and observed (squares) spectra of the two Nd II lines at 4012.24 and $4012.69 \AA$.

Gaussian profile to the observed lines of interest using the interactive splot function in the $\mathrm{IRAF}^{4}$ package. All $\mathrm{Zr}$ and $\mathrm{Ba}$ abundances were obtained by $\mathrm{EW}$ analysis, while the $\mathrm{La}, \mathrm{Ce}$, and $\mathrm{Nd}$ lines required syntheses. A sample synthesis of $\mathrm{Nd}$ lines is shown in Figure 1.

Our analysis produced absolute abundances; but, to answer the question of chemical homogeneity in the Hyades, we use differential abundances. The final differential abundances $\Delta[X / \mathrm{H}]$ were derived by subtracting the absolute abundance of each individual line of the reference star vB 153 from the same line in the sample stars and then taking the mean of the differences for each element. The star vB 153 was chosen for reference because it was the reference star used in the differential abundance work by $\mathrm{P} 03$, hence enabling easy comparisons. By using such a lineby-line differential technique, we also reduce the errors due to the uncertainty in the line data, hence minimizing the star-to-star scatter. The differential abundances of all elements are plotted in Figure 2. The absolute abundances for all stars are given in Table 2.

\subsection{Error Analysis}

The main sources of error in the present study are EW measurements, continuum placement, and stellar parameters. External errors, such as uncertainties in the line data and model atmospheres, are the smallest sources of error, since we are interested only in the differential abundances. The number of lines used to calculate the final abundances also contributed to the total uncertainty for each element.

The error in EWs estimated by repeated measurements of each line is between 0.5 and $5 \mathrm{~m} \AA$, depending on the strength of the lines, corresponding to abundance errors of $0.01-0.05 \mathrm{dex}$. The measurement errors for the synthesized abundances are derived by changing the abundance until there is a clear visible deviation from the best fit. The differential errors in the stellar parameters were assumed to be $\delta T_{\text {eff }}=50 \mathrm{~K}, \delta \log g=0.1 \mathrm{~cm} \mathrm{~s}^{-2}$, and $\delta \xi=$ $0.1 \mathrm{~km} \mathrm{~s}^{-1}$. Table 3 shows the abundance dependencies on the stellar parameters. Typical values of the total estimated uncertainty

\footnotetext{
${ }^{4}$ IRAF is distributed by the National Optical Astronomy Observatory, which is operated by the Association of Universities for Research in Astronomy, Inc., under cooperative agreement with the National Science Foundation.
} 

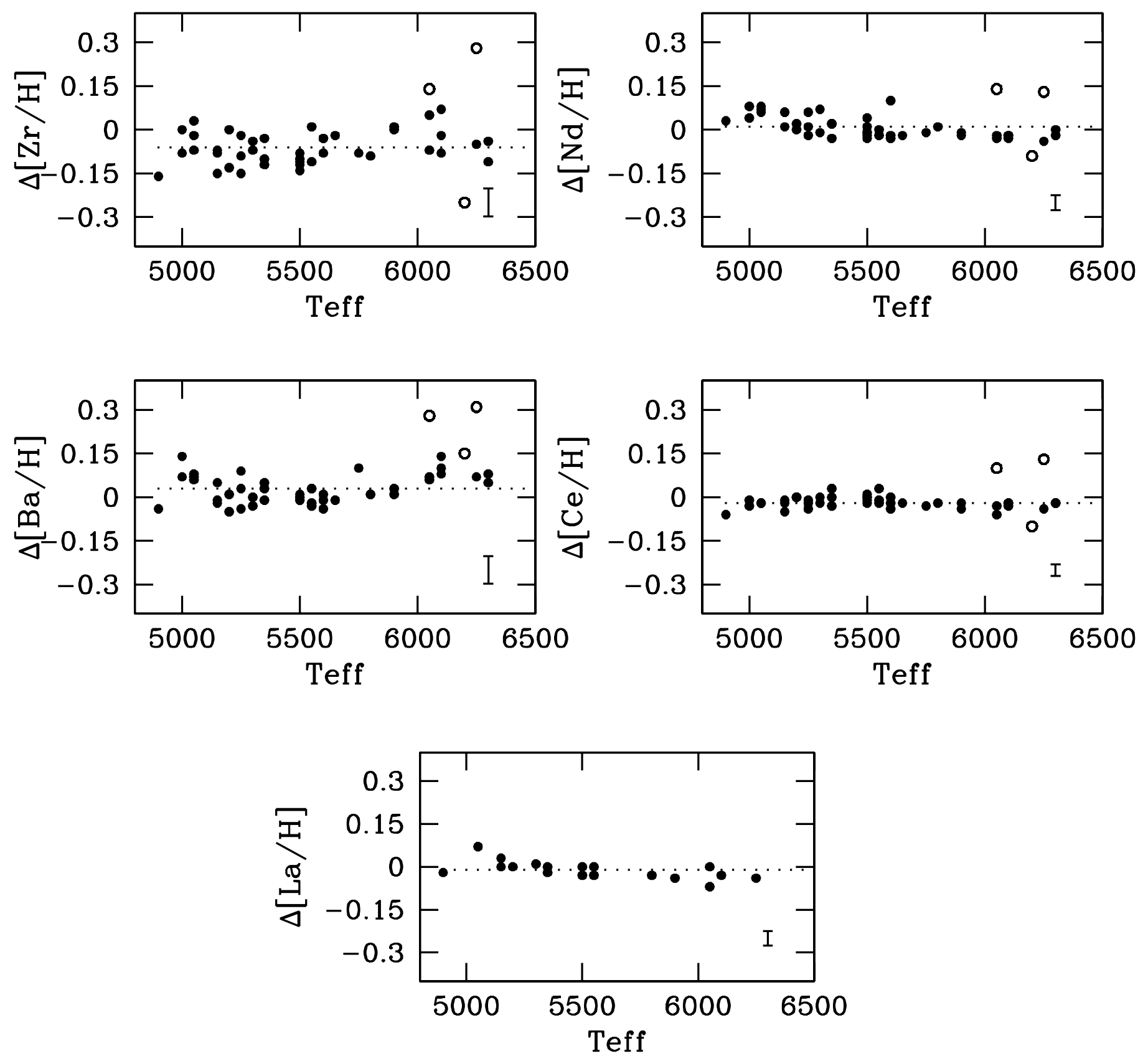

FIg. 2.-Differential $[X / \mathrm{H}]$ vs. effective temperature. The differential comparison star is vB 153 , as discussed in the text. The open symbols represent stars believed to be nonmembers, as discussed in $\S 4$.2. The dotted lines represent mean abundance levels. The error bars indicate the typical abundance uncertainty for each element.

in each element are as follows: $\mathrm{Zr}=0.048, \mathrm{Ba}=0.047, \mathrm{La}=$ $0.025, \mathrm{Ce}=0.025$, and $\mathrm{Nd}=0.026 \mathrm{dex}$.

\section{ABUNDANCE TRENDS}

The final results show uniform abundances, although some elements show a small residual trend with temperature. Slightly increasing slopes are seen for $\mathrm{Zr}$, while decreasing trends are seen for La and $\mathrm{Nd}$. It is very likely that these trends are a systematic effect resulting from inadequacies in the model atmospheres and do not reflect intrinsic abundance variations of the Hyades.

\subsection{Stellar Parameters}

The abundances for the $\alpha$ and Fe-peak elements by P03 show no trends such as those seen here for the neutron-capture elements (except for $\mathrm{Ca}$, which has an increasing slope with temperature thought to be due to saturating $\mathrm{Ca}$ lines in the cooler stars). Since we have used the same stellar parameters, it is unlikely that errors in the parameters caused the currently observed trends. Furthermore, any change in the model parameters will not correct all the trends, as different elements show trends in opposite directions. As a check, each parameter $\left(T_{\text {eff }}, \log g\right.$, and microturbulence) was varied in an attempt to remove the trends. For effective temperature, the coolest stars were made cooler and the hottest stars made hotter by $\delta T_{\text {eff }}=100 \mathrm{~K}$. Similarly, the surface gravity was varied by $\delta \log g=0.1 \mathrm{~cm} \mathrm{~s}^{-2}$ and microturbulence by $\delta \xi=0.1 \mathrm{~km} \mathrm{~s}^{-1}$. Such changes will flatten the slopes for La and $\mathrm{Nd}$, while the same changes increase the slopes of the $\mathrm{Ba}$ and $\mathrm{Zr}$ abundances.

Also, to further confirm our method of analysis, we reanalyzed the $\mathrm{Mg}$ abundances for a subsample of stars covering the 
TABLE 2

Elemental Abundances

\begin{tabular}{|c|c|c|c|c|c|c|c|}
\hline HD & Other Name & {$[\mathrm{Fe} / \mathrm{H}]$} & {$[\mathrm{Zr} / \mathrm{H}]$} & {$[\mathrm{Ba} / \mathrm{H}]$} & {$[\mathrm{La} / \mathrm{H}]$} & {$[\mathrm{Ce} / \mathrm{H}]$} & {$[\mathrm{Nd} / \mathrm{H}]$} \\
\hline $28635 \ldots \ldots \ldots \ldots$ & vB 88 & 0.09 & 2.68 & 2.67 & 1.15 & 1.64 & 1.41 \\
\hline $26257 \ldots \ldots \ldots \ldots$ & HIP 19386 & 0.11 & 2.62 & 2.65 & $\ldots$ & 1.59 & 1.43 \\
\hline $35768 \ldots \ldots \ldots \ldots$ & HIP 25639 & 0.08 & 2.69 & 2.68 & $\ldots$ & 1.65 & 1.45 \\
\hline $20430 \ldots \ldots \ldots \ldots$ & vB 1 & 0.30 & 3.01 & 2.91 & $\ldots$ & 1.85 & 1.58 \\
\hline $29419 \ldots \ldots \ldots \ldots \ldots$ & vB 105 & 0.13 & 2.65 & 2.70 & 1.16 & 1.67 & 1.43 \\
\hline $30589 \ldots \ldots \ldots \ldots$ & vB 118 & 0.15 & 2.71 & 2.68 & $\ldots$ & 1.68 & 1.42 \\
\hline $27835 \ldots \ldots \ldots \ldots$ & vB 49 & 0.09 & 2.66 & 2.66 & 1.19 & 1.69 & 1.43 \\
\hline $25825 \ldots \ldots \ldots \ldots . . . .$. & vB 10 & 0.15 & 2.80 & 2.74 & $\ldots$ & 1.76 & 1.43 \\
\hline $27859 \ldots \ldots \ldots \ldots$ & vB 52 & 0.14 & 2.78 & 2.67 & 1.12 & 1.69 & 1.42 \\
\hline $20439 \ldots \ldots \ldots \ldots$ & vB 2 & 0.30 & 2.87 & 2.88 & $\ldots$ & 1.84 & 1.59 \\
\hline $28992 \ldots \ldots \ldots \ldots . . . . . . .$. & vB 97 & 0.12 & 2.73 & 2.61 & $\ldots$ & 1.67 & 1.43 \\
\hline $26767 \ldots \ldots \ldots \ldots$ & vB 18 & 0.12 & 2.74 & 2.63 & 1.17 & 1.71 & 1.44 \\
\hline $26736 \ldots \ldots \ldots \ldots . . .$. & vB 15 & 0.09 & 2.65 & 2.70 & $\ldots$ & 1.74 & 1.44 \\
\hline 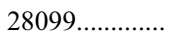 & vB 64 & 0.10 & 2.64 & 2.61 & 1.16 & 1.74 & 1.46 \\
\hline $26756 \ldots \ldots \ldots \ldots$ & vB 17 & 0.06 & 2.71 & 2.59 & $\ldots$ & 1.74 & 1.43 \\
\hline $27282 \ldots \ldots \ldots \ldots$ & vB 27 & 0.15 & 2.70 & 2.61 & $\ldots$ & 1.78 & 1.42 \\
\hline $240648 \ldots \ldots \ldots \ldots$ & HIP 23750 & 0.15 & 2.65 & 2.59 & $\ldots$ & 1.76 & 1.43 \\
\hline $19902 \ldots \ldots \ldots \ldots . . . . . .$. & HIP 14976 & 0.09 & 2.70 & 2.56 & $\ldots$ & 1.73 & 1.55 \\
\hline $28593 \ldots \ldots \ldots \ldots . . .$. & vB 87 & 0.11 & 2.62 & 2.57 & 1.16 & 1.75 & 1.43 \\
\hline $31609 \ldots \ldots \ldots \ldots$ & vB 127 & 0.15 & 2.74 & 2.63 & 1.19 & 1.78 & 1.45 \\
\hline 26015B ........... & vB 12 & 0.17 & 2.58 & 2.63 & $\ldots$ & 1.82 & 1.41 \\
\hline $28805 \ldots \ldots \ldots \ldots . . .$. & vB 92 & 0.08 & 2.59 & 2.59 & $\ldots$ & 1.73 & 1.44 \\
\hline $27250 \ldots \ldots \ldots \ldots$ & vB 26 & 0.09 & 2.65 & 2.58 & $\ldots$ & 1.75 & 1.46 \\
\hline $27732 \ldots \ldots \ldots \ldots$ & vB 42 & 0.10 & 2.63 & 2.60 & 1.16 & 1.76 & 1.44 \\
\hline $32347 \ldots \ldots \ldots \ldots . .$. & vB 187 & 0.11 & 2.63 & 2.61 & 1.19 & 1.77 & 1.42 \\
\hline $242780 \ldots \ldots \ldots \ldots$ & HIP 24923 & 0.12 & 2.62 & 2.60 & $\ldots$ & 1.76 & 1.43 \\
\hline $283704 \ldots \ldots \ldots . . .$. & vB 76 & 0.09 & 2.61 & 2.59 & $\ldots$ & 1.78 & 1.49 \\
\hline $284574 \ldots \ldots \ldots \ldots$ & vB 109 & 0.13 & 2.63 & 2.59 & 1.17 & 1.74 & 1.42 \\
\hline $284253 \ldots \ldots \ldots \ldots$ & vB 21 & 0.14 & 2.70 & 2.63 & $\ldots$ & 1.81 & 1.47 \\
\hline $285773 \ldots \ldots \ldots . .$. & vB 79 & 0.14 & 2.69 & 2.57 & 1.20 & 1.76 & 1.52 \\
\hline $30505 \ldots \ldots \ldots \ldots$ & vB 116 & 0.13 & 2.66 & 2.60 & $\ldots$ & 1.78 & 1.44 \\
\hline \multirow[t]{3}{*}{$28258 \ldots \ldots \ldots \ldots$} & vB 178 & 0.15 & 2.61 & 2.65 & 1.19 & 1.80 & 1.47 \\
\hline & vB 4 & 0.15 & 2.71 & 2.69 & $\ldots$ & 1.86 & 1.51 \\
\hline & vB 153 & 0.10 & 2.73 & 2.61 & 1.19 & 1.90 & 1.45 \\
\hline $27771 \ldots \ldots \ldots \ldots$ & vB 46 & 0.08 & 2.60 & 2.55 & $\ldots$ & 1.81 & 1.47 \\
\hline $28462 \ldots \ldots \ldots \ldots$ & vB 180 & 0.08 & 2.64 & 2.56 & $\ldots$ & 1.75 & 1.46 \\
\hline $29159 \ldots \ldots \ldots \ldots . .$. & vB 99 & 0.09 & 2.65 & 2.67 & $\ldots$ & 1.87 & 1.49 \\
\hline $28878 \ldots \ldots \ldots \ldots . . .$. & vB 93 & 0.13 & 2.65 & 2.65 & 1.19 & 1.83 & 1.46 \\
\hline $285367 \ldots \ldots \ldots \ldots$ & HIP 19098 & 0.11 & 2.58 & 2.59 & $\ldots$ & 1.82 & 1.51 \\
\hline \multirow[t]{2}{*}{$285252 \ldots \ldots \ldots \ldots$} & vB 7 & 0.15 & 2.71 & 2.66 & $\ldots$ & 1.85 & 1.51 \\
\hline & vB 5 & 0.16 & 2.66 & 2.68 & $\ldots$ & 1.84 & 1.52 \\
\hline $28977 \ldots \ldots \ldots \ldots . . . . .$. & vB 183 & 0.13 & 2.66 & 2.58 & 1.22 & 1.80 & 1.51 \\
\hline $18632 \ldots \ldots \ldots \ldots$ & HIP 13976 & 0.18 & 2.73 & 2.74 & $\ldots$ & 1.86 & 1.53 \\
\hline $285830 \ldots \ldots \ldots \ldots$ & vB 179 & 0.22 & 2.76 & 2.67 & 1.26 & 1.94 & 1.53 \\
\hline $285690 \ldots \ldots \ldots . .$. & vB 25 & 0.08 & 2.57 & 2.56 & 1.15 & 1.77 & 1.48 \\
\hline $14127 \ldots \ldots \ldots \ldots$ & HIP 10672 & -0.12 & 2.48 & 2.75 & $\ldots$ & 1.58 & 1.36 \\
\hline
\end{tabular}

TABLE 3

Abundance Dependencies on Model Parameters

\begin{tabular}{|c|c|c|c|c|c|}
\hline Model Parameter & $\delta[\mathrm{Zr} / \mathrm{H}]$ & $\delta[\mathrm{Ba} / \mathrm{H}]$ & $\delta[\mathrm{La} / \mathrm{H}]$ & $\delta[\mathrm{Ce} / \mathrm{H}]$ & $\delta[\mathrm{Nd} / \mathrm{H}]$ \\
\hline \multicolumn{6}{|c|}{ vB $25\left(T_{\text {eff }}=4900\right)$} \\
\hline$T_{\text {eff }} \pm 50(\mathrm{~K}) \ldots \ldots \ldots \ldots$ & \pm 0.01 & \pm 0.02 & \pm 0.01 & \pm 0.01 & \pm 0.02 \\
\hline $\log g \pm 0.1 \ldots \ldots \ldots \ldots \ldots$ & \pm 0.03 & 0.00 & \pm 0.01 & 0.00 & 0.00 \\
\hline$\xi \pm 0.1\left(\mathrm{~km} \mathrm{~s}^{-1}\right) \ldots \ldots$. & $\mp 0.02$ & $\mp 0.03$ & \pm 0.02 & 0.00 & \pm 0.02 \\
\hline \multicolumn{6}{|c|}{$\mathrm{vB} 105\left(T_{\mathrm{eff}}=6100\right)$} \\
\hline$T_{\text {eff }} \pm 50(\mathrm{~K}) \ldots \ldots \ldots \ldots$ & \pm 0.01 & \pm 0.02 & \pm 0.01 & \pm 0.01 & 0.00 \\
\hline $\log g \pm 0.1 \ldots \ldots \ldots \ldots \ldots \ldots$ & \pm 0.04 & \pm 0.01 & 0.00 & \pm 0.01 & \pm 0.02 \\
\hline$\xi \pm 0.1\left(\mathrm{~km} \mathrm{~s}^{-1}\right) \ldots \ldots$. & $\mp 0.02$ & $\mp 0.03$ & \pm 0.01 & 0.00 & 0.00 \\
\hline
\end{tabular}




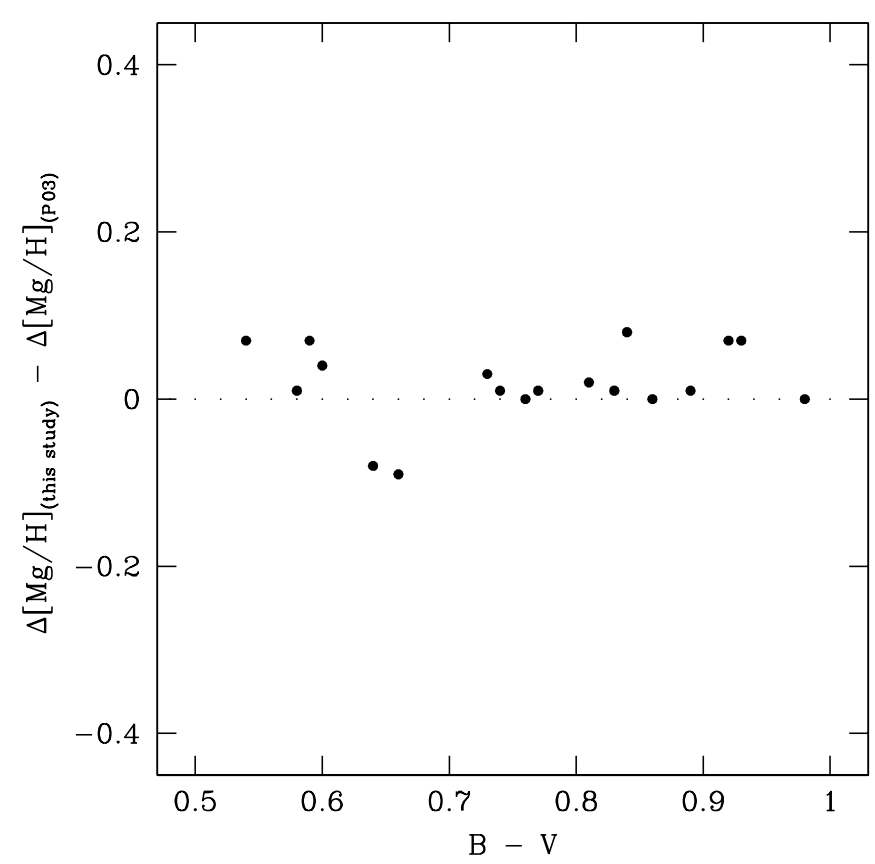

Fig. 3.-Comparison of $\mathrm{Mg}$ abundances with $\mathrm{P} 03$ for a subsample of stars covering the full temperature range. The mean of the difference $\Delta[\mathrm{Mg} / \mathrm{H}]_{\text {this study }}-$ $\Delta[\mathrm{Mg} / \mathrm{H}]_{\mathrm{P} 03}$ is 0.02 dex, with a standard deviation of $0.03 \mathrm{dex}$; but, most importantly, no slope is present.

full temperature range and successfully recovered the result of $\mathrm{P} 03$, which does not show any trends. A comparison plot is shown in Figure 3. The mean of the difference $\Delta[\mathrm{Mg} / \mathrm{H}]_{\text {this study }}-$ $\Delta[\mathrm{Mg} / \mathrm{H}]_{\mathrm{P} 03}=0.02 \mathrm{dex}$, with a standard deviation of 0.03 dex.

\subsection{Continuum Placement}

Note that the scatter for all elements increases with lower temperature. As the temperature decreases, the greater the blending and therefore the harder the continuum placement. Since most lines lie in the blue part of the spectral region, which is highly crowded, determining the continuum level is difficult. It is possible that as the lines begin to blend in the cooler stars, the continuum is placed too low, and a lower abundance is estimated for the cooler stars in comparison to the stars at the hotter end. Such an effect would explain the increasing trends observed for $\mathrm{Zr}$, but we cannot explain the decreasing slopes as due to an error in continuum placement.

\subsection{Hyperfine Structure}

Many of the heavy-element lines are affected by hyperfine structure. In most cases the effects are small enough to be negligible, especially in very weak lines. However, as lines saturate and move off the linear part of the curve of growth, these effects become more important. If an observed line is made up of many components that are greater than the intrinsic width of the line as calculated by standard means of broadening (thermal, microturbulence, etc.), then neglecting the splitting would result in overestimating the abundance value. The transition lines for $\mathrm{La}$, which has widely known hyperfine structure, have been synthesized accordingly. However, a slight slope is still present in the La results after accounting for the hyperfine structure.

\subsection{Non-LTE Effects}

Finally, we suspect that non-LTE (NLTE) effects may play a role in these abundance trends. NLTE effects are specific to the
TABLE 4

Regression Line Parameters

\begin{tabular}{|c|c|c|}
\hline Element & $\alpha$ & $\beta$ \\
\hline 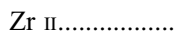 & 2.88 & -0.22 \\
\hline $\mathrm{Ba}$ II .................... & 2.81 & -0.19 \\
\hline 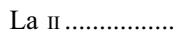 & 0.90 & 0.29 \\
\hline $\mathrm{Ce}$ II ...................... & 1.72 & 0.04 \\
\hline Nd II ..................... & 1.11 & 0.37 \\
\hline
\end{tabular}

atom and affect each element differently rather than generally (cf. stellar parameters). Since we observe slopes in opposite directions, it is very likely that we are seeing NLTE effects. Unfortunately, there have not been many NLTE calculations done for the heavier neutron-capture elements in order to determine what such effects would be. Mashonkina et al. (1999) find that for the $\mathrm{Ba}$ II line at $5853 \AA$ the correction is less than $0.1 \mathrm{dex}$; however, it does not cover the full range of temperatures we are covering in this analysis. We are more interested to see whether NLTE effects come into play over a large range of temperatures in order to explain the observed abundance trends. We are not aware of any published work that indicates such NLTE effects.

A study of NLTE effects for heavy elements is beyond the scope of this paper; but, in an attempt to quantify this possible NLTE correction, we have fitted least-squares regression lines to the observed trends. The fits are in the form of $\Delta[X / \mathrm{H}]=$ $\alpha+\beta\left(5200 \mathrm{~K} / T_{\text {eff }}\right)$, where $T_{\text {eff }}$ has been normalized by the $T_{\text {eff }}$ of the reference star $\mathrm{vB} 153$. The values of $\alpha$ and $\beta$ are given for each element in Table 4. We look forward to future NLTE calculations that may explain the currently observed abundance trends.

\section{DISCUSSION}

\subsection{Chemical Homogeneity}

With regards to chemical homogeneity and establishing chemical signatures for the Hyades, our results are positive. The tightly correlated abundances are an indication of the level of homogeneity that exists within the cluster. The observed scatter for the Hyades members $\sigma_{\mathrm{obs}}$, the estimates of the intrinsic scatter $\sigma_{\mathrm{int}}$, and the corresponding $\chi^{2}$ values are given in Table 5. The value of $\sigma_{\text {int }}$ was estimated using the equations $\sigma_{\text {obs }}^{2}=\sigma_{\text {int }}^{2}+\sigma_{\text {expected }}^{2}$ and $\chi_{r}^{2}=\sigma_{\text {obs }}^{2} / \sigma_{\text {expected, }}^{2}$, where $\sigma_{\text {expected }}$ is the estimated uncertainty as discussed in $\S 2.4$. With the estimated uncertainties being typically around $\sigma_{\mathrm{obs}},\left\langle\chi_{r}^{2}\right\rangle \approx 1$. This implies that the total star-to-star scatter is within the measurable limit and that the true intrinsic scatter among the Hyades stellar members is extremely low.

This level of chemical uniformity is observational evidence for a chemically well-mixed gas cloud. It is possible that these dwarfs formed later, after the high-mass stars evolved to produce several supernovae, which enriched the gas as well as contributing to the mixing of the gas cloud. Although the actual

TABLE 5

Abundance Scatter

\begin{tabular}{|c|c|c|c|}
\hline Element & $\sigma_{\mathrm{obs}}$ & $\sigma_{\text {int }}$ & $\chi_{r}^{2}$ \\
\hline $\mathrm{Zr} \ldots \ldots \ldots \ldots \ldots \ldots$ & 0.055 & 0.026 & 1.30 \\
\hline $\mathrm{Ba}$ & 0.049 & 0.013 & 1.08 \\
\hline $\mathrm{La}$ & 0.025 & 0.000 & 1.00 \\
\hline $\mathrm{Ce}$ & 0.025 & 0.000 & 1.00 \\
\hline 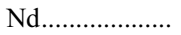 & 0.032 & 0.018 & 1.51 \\
\hline
\end{tabular}


mechanism for the mixing is still debatable, it is clear from our results that the gas cloud was uniformly mixed by the time these dwarf stars formed.

Also, the detected levels of chemical homogeneity indicate that these stars have not been polluted either from local sources (such as stellar winds or supernova explosions) or from sweptup gas from the interstellar medium. Quillen (2002) derived an upper limit of 0.03 dex for the star-to-star metallicity scatter using the Hyades H-R diagram, indicating that pollution effects are neither common nor strong. Our estimated $\sigma_{\text {int }}$ for the heavier elements is within this upper limit.

\subsection{Stellar Membership}

A further reason to interpret the results as a strong case for homogeneity and a positive sign of establishing a chemical signature is the deviation of a few stars that are thought to be nonmembers found in the same direction as the cluster. The two stars vB 1 and $\mathrm{vB} 2$ are clearly overabundant by more than $2 \sigma$ in all elements. P03 also found these stars to be significantly enriched. These stars were earlier considered to be Hyades members, as they have consistent photometry, radial velocities, and Hipparcos distances for membership; however, de Bruijne et al. (2001) find that they are nonmembers based on proper motions and trigonometric parallax measurements. The star HD 14127 is underabundant in $\mathrm{Zr}, \mathrm{Ce}, \mathrm{La}$, and $\mathrm{Nd}$; however, it is slightly overabundant in Ba. P03 classify it as a nonmember due to its metallicity being -0.25 dex below the cluster mean, its Hipparcos distance being too large, and its location being below the Hyades main sequence.

\subsection{Implications for Chemical Tagging}

Since most of the dynamical information on the Galactic disk has been subject to evolutionary processes of dissipation and scattering, chemical information locked within the stars provides the only true tracer left of initial identity. Having established the Hyades open cluster members to be homogeneous in the heavier neutron-capture elements, along with the $\alpha$ and Fe-peak elements (P03), as well as the fact that several nonmembers deviate from the cluster mean, supports the case for using chemical abundances as tracers of cluster membership and establishing chemical signatures unique to the stellar members of the cluster. We are more confident now that chemical information can be used to identify the larger star-forming aggregates in the early disk by means of chemical tagging.

This paves the way for chemically tagging other member stars that are at present no longer part of the bound Hyades system. A necessary test would be to study the stars of the Hyades supercluster, which is thought to be a moving stellar group (see Eggen 1970). It will be very interesting indeed to check whether the same abundance patterns are seen among the stellar members of the supercluster as are seen in the core of the Hyades open cluster we have presented here. Such a test will be the next step forward in truly demonstrating the viability of chemical tagging for the future.

This research has made use of the Vienna Atomic Line Database (VALD), operated at Vienna, Austria, and the Database on Rare Earths At Mons University (DREAM), operated at Mons, Belgium.
Beckers, J. M., Bridges, C. A., \& Gilliam, L. B. 1976, A High Resolution Spectral Atlas of the Solar Irradiance from 380 to 700 Nanometers, Volume 2: Graphical Form (Hanscom AFB: Air Force Geophys. Lab)

Bland-Hawthorn, J., \& Freeman, K. C. 2004, Publ. Astron. Soc. Australia, 21, 110

Burbidge, E. M., Burbidge, G. R., Fowler, W. A., \& Hoyle, F. 1957, Rev. Mod. Phys., 29, 547

Castelli, F., Gratton, R. G., \& Kurucz, R. L. 1997, A\&A, 318, 841

Castro, S., Porto de Mello, G. F., \& da Silva, L. 1999, MNRAS, 305, 693

Cochran, W. D., Hatzes, A. P., \& Paulson, D. B. 2002, AJ, 124, 565

Conti, P. S., Wallerstein, G., \& Wing, R. F. 1965, ApJ, 142, 999

de Bruijne, J. H. J., Hoogerwerf, R., \& de Zeeuw, P. T. 2001, A\&A, 367, 111

Den Hartog, E. A., Lawler, J. E., Sneden, C., \& Cowan, J. J. 2003, ApJS, 148, 543

Edvardsson, B., Andersen, J., Gustafsson, B., Lambert, D. L., Nissen, P. E., \& Tomkin, J. 1993, A\&A, 275, 101

Eggen, O. J. 1970, PASP, 82, 99

Freeman, K., \& Bland-Hawthorn, J. 2002, ARA\&A, 40, 487

Friel, E. D., Jacobson, H. R., Barrett, E., Fullton, L., Balachandran, S. C., \& Pilachowski, C. A. 2003, AJ, 126, 2372

Gonzalez, G., \& Wallerstein, G. 2000, PASP, 112, 1081

Hoogerwerf, R., \& Aguilar, L. A. 1999, MNRAS, 306, 394

\section{EFERENCES}

Kupka, F., Piskunov, N. E., Ryabchikova, T. A., Stempels, H. C., \& Weiss, W. W. 1999, A\&AS, 138, 119

Lawler, J. E., Bonvallet, G., \& Sneden, C. 2001, ApJ, 556, 452

Mashonkina, L., Gehren, T., \& Bikmaev, I. 1999, A\&A, 343, 519

McKee, C. F., \& Tan, J. C. 2002, Nature, 416, 59

Palmeri, P., Quinet, P., Wyart, J.-F., \& Biémont, E. 2000, Phys. Scr., 61, 323

Paulson, D. B., Sneden, C., \& Cochran, W. D. 2003, AJ, 125, 3185 (P03)

Perryman, M. A. C., et al. 1998, A\&A, 331, 81

Piskunov, N. E., Kupka, F., Ryabchikova, T. A., Weiss, W. W., \& Jeffery, C. S. 1995, A\&AS, 112, 525

Quillen, A. C. 2002, AJ, 124, 400

Reddy, B. E., Tomkin, J., Lambert, D. L., \& Allende Prieto, C. 2003, MNRAS, 340,304

Ryabchikova, T. A., Piskunov, N. E., Stempels, H. C., Kupka, F., \& Weiss, W. W. 1999, Phys. Scr., T83, 162

Schuler, S. C., King, J. R., Fischer, D. A., Soderblom, D. R., \& Jones, B. F. 2003, AJ, 125, 2085

Sneden, C. A. 1973, Ph.D. thesis, Univ. Texas

Tautvaišiene, G., Edvardsson, B., Tuominen, I., \& Ilyin, I. 2000, A\&A, 360, 499

Wallerstein, G., et al. 1997, Rev. Mod. Phys., 69, 995 